Journal of Advanced Research in Fluid Mechanics and Thermal Sciences

Journal homepage: www.akademiabaru.com/arfmts.html ISSN: $2289-7879$

\title{
Dean Vortex for Laminar Flows in Curved Pipes with Various Cross-Sections
}

\author{
Siham Ajgoun ${ }^{1,}{ }^{*}$, Jaafar Khalid Naciri ${ }^{1}$, Rabha Khatyr ${ }^{1}$ \\ 1 Laboratory of Mechanics, Faculty of Sciences Aïn Chock, B.P. 5366, Maarif, Hassan II University, Casablanca 20100, Morocco
}

\begin{tabular}{|c|c|}
\hline ARTICLE INFO & ABSTRACT \\
\hline $\begin{array}{l}\text { Article history: } \\
\text { Received } 2 \text { July } 2020 \\
\text { Received in revised form } 22 \text { August } 2020 \\
\text { Accepted } 24 \text { August } 2020 \\
\text { Available online } 31 \text { December } 2020\end{array}$ & $\begin{array}{l}\text { Curved pipes flows are encountered in different areas such as heat transfer, chaotic } \\
\text { mixing, separation of mixtures in pipes, or blood circulation among others and exhibit } \\
\text { a variety of characteristics depending on the ranges of Dean numbers and pipe } \\
\text { curvatures. Studies on curved pipes flows usually consider the cases of circular, } \\
\text { elliptical, and rectangular shapes for the cross sections of the pipe. The present work } \\
\text { extends the availability of asymptotic analytical solutions to new ranges of cross- } \\
\text { sectional shapes while considering fully developed steady state flows at low Dean } \\
\text { numbers. The new shapes are given by a polar equation } R^{*}(\theta) \text { satisfying the relation } \\
1-R^{* 2}(\theta)+\delta R^{* \gamma}(\theta) \sin (\gamma \theta)=0 \text { where } \delta \text { and } \gamma \text { are parameters. The zero-order } \\
\text { azimuthal velocity profiles for various cross-sections are given by exact analytical } \\
\text { solutions. Solutions for the non-homogeneous biharmonic equation for the secondary } \\
\text { flows are given by using exact expressions for the particular solutions. Furthermore, } \\
\text { the Fourier series decomposition of the solution is adopted to determine the } \\
\text { integration constants that allow satisfying the non-slip boundary conditions. Solutions } \\
\text { are presented for semi triangular }(\gamma=3) \text {, square }(\gamma=4) \text {, and pentagonal }(\gamma=5) \\
\text { cross sections shapes. It is found that the velocity distribution and the Dean's vortexes } \\
\text { intensities are modified in function of the cross-section shapes. }\end{array}$ \\
\hline
\end{tabular}

Keywords:

Dean vortex; Curved pipes; Various cross-sections; Biharmonic equation; Method of fundamental solutions

Copyright $@ 2021$ PENERBIT AKADEMIA BARU - All rights reserved

\section{Introduction}

Laminar fluid flow in curved pipes with a slowly varying radius of curvature and for various crosssections shapes is encountered in different areas such as heat transfer [1], chaotic mixing [2], separation of mixtures in pipes [3], or blood circulation in the arteries [4] among others. In inertial microfluidic technology, many applications use the properties of Dean's vortices to manipulate the motion of small particles at low cost based solely on the hydrodynamic properties of these flows, a detailed review of the use of secondary flows in microfluidics was recently presented by Zhao et al., [5]. For applications based on inertial focusing separation, cross-sections shapes are essentials for

\footnotetext{
* Corresponding author.

E-mail address: s.ajgoun@outlook.fr

https://doi.org/10.37934/arfmts.79.1.111127
} 
mastering the separation, which is generally carried out in spiral tubes, thus with small variations in the radius of curvature [6]. Guan et al., [7], by using a new method for three-dimensional observation of the location of focused particle stream, introduced a novel spiral microchannel with a trapezoidal cross-section that generates stronger Dean vortices at the outer half of the channel and achieved higher separation resolution than conventional spiral microchannels with rectangular cross-section. Thus, by acting on the shape of the pipe, one can modify the flow characteristics and, modulate the shape and intensity of the secondary flows which appear in the curved conduits. The control of the induced modifications makes it possible to optimize the yields for the envisaged uses. This broad potential for use motivated many studies on flows in curved pipes in various situations [8].

Curved pipe flows exhibit a variety of characteristics depending on the ranges of Reynolds numbers and pipe curvatures considered for the intended fields of application. The parameters governing such flows are fixed by the ratios of orders of magnitude between inertial, centrifugal, and viscous forces. The main feature of curved pipe flows is the presence of centrifugal forces which induce secondary flows, known as Dean's vortex. These vortices are characterized by the Dean number, defined as the ratio between orders of magnitude of the centrifugal forces and the part of the viscous forces acting perpendicular to the main direction of the flow, and which is related to flow patterns according to the range of its values. Regarding the fluid dynamics aspect, a first classification of curved pipe flows into three large categories, i.e. steady state, periodic and chaotic, according to the Dean number values, has been proposed by Yanase et al., [9]. Thus for industrial applications in the field of heat transfer, depending on the values taken by the Grashof number, it will be necessary to take into account the variation in density of the fluid, as in Mondal et al., [10] and even to consider variable physical fluid properties while for other range of values these effects will be weak and one will consider the fluid as incompressible with constant physical properties. For applications in microfluidics, such as for equipment for biological kits or again for cooling techniques in small spaces among others, the small dimensions of the channels mean that the Reynolds numbers remain moderate and therefore the flows are at low inertia [11]. In the latter cases, low Dean number approaches may prove to be suitable, the problem is then generally more focused on the cross sectional shapes of the pipe, the aspect ratios and in some cases on the non-Newtonian character of the fluid as in the case for example of blood flows in parts of the arterial network [12]. The present work, rather of a mathematical nature and based on asymptotic expansions valid for low Dean numbers, is to be classified in the category of low Dean numbers steady state 2D curved flows studies, it aims to explores the possibility of giving analytical or semi-analytical solutions, adapted to a wide range of non-circular geometries. Following the example of the work proposed by Norouzi et al., [11] for rectangular cross-section conduits based on asymptotic expansions with respect to the curvature parameter, and which deals in detail with the limits of validity of the asymptotic approaches, we propose, by using similar but not identical approaches, solutions for a wide range of shapes of the generic cross-sections of the tube.

Recall that the first solutions establishing the existence of secondary flows in curved pipes with circular cross-sections are those obtained analytically by Dean [13-14] in the form of asymptotic expansions valid for small values of the Dean number. The presence of secondary flows will be confirmed experimentally by Eustice [15-16]. Subsequently, Cumming [17] in 1957 presented asymptotic solutions for elliptical and square cross-section conduits, and Ito [18] discussed solutions in the case of rectangular cross-sections.

Berger and Talbot [8] presented a review in 1983 of curved pipe flow studies from Dean's early analytic and asymptotic results to the most recent ones obtained by finite difference numerical methods [19] available at this stage. This document recalls the mathematical formulation to be retained in the case of fully developed flows as well as the asymptotic expansions to be adopted in 
cases of low Dean number values and reminds moreover that the effect of secondary flows on the pressure drop is obtained considering the second-order terms in Dean's developments in the case of torus with circular cross-sections.

Starting from the theoretical configuration of a torus, one can introduce extended geometric properties to integrate other features such as variable curvature or torsion. Wang [20] in 1981, have used a non-orthogonal helical coordinate system to study the impact of curvature and torsion on the flow in a helical pipe. They noticed that when the torsion becomes more marked, the secondary flow develops as a single circulating cell, and the changes in curvature affect only the flow rate along the pipe. Germano [21] in 1982 introduced an orthogonal coordinate system to study the effect of curvature and torsion on flow in a helical pipe. Gammack and Hydon [22] in 2001 solved numerically and analytically the steady and unsteady flows for four types of curved pipe with non-uniform curvatures and torsions. They considered that the curvature $k$ is an exponential function of the azimuthal coordinate $\mathrm{s}\left(k=k_{0} e^{\eta \mathrm{s}}\right.$, with $k_{0}$ and $\eta$ are constants). They showed that the elevation of the curvature in $s$ produce an increase of velocity amplitude in the cross-section, and the maximum axial velocity is forced near to the wall.

In addition, curved pipes flows are widely studied within the framework of the modeling of the blood flows in the arterial network, with a view to a better understanding of the relations between the dynamics of flow and pathologies such as the case of arteriosclerosis, or even for the development of non-invasive diagnostic aid techniques. In 1980, Pedley [23] gave a general overview of the flows in curved large blood vessels (heart, arteries, and veins). Singers [4] in 2005 focused his research on blood circulation in a curved artery. They analyzed the impact of centrifugal and Coriolis forces on flows in pipes of finite curvature. Zakaria et al., [24] in 2018 presented the results obtained by $3 \mathrm{D}$ numerical simulation using the computational fluid dynamics platform OpenFOAM for the study of blood flow in the human aorta. They focused on complex flow in the curved tube like secondary and separation flows due to their impact on the wall shear stress.

Other studies deal with instabilities and unsteadiness for curved pipes flows. Fellouah et al., [25] in 2006 presented a numerical and experimental study of Dean instability for the flow in curved pipes. Timite et al., [26] in 2010 examined numerically and experimentally the effects of the pulsed flow on the secondary flow in a curved pipe. Mondal et al., [10] studied numerically the fully developed twodimensional unsteady flow of viscous incompressible fluid through a curved rectangular duct by using a spectral method for a range of Dean numbers between 1000 and 10000 and investigate the nonlinear behavior of the unsteady solutions. They showed that convective heat transfer is significantly improved by the secondary flow and the chaotic flow, which occurs at large Dean's number, enhances heat transfer more strongly than the steady-state or periodic solutions. In relation with the growing interest on engineering applications involving rotating coiled ducts, recent works by Islam et al., [27] studied the effects of rotation on the unsteady flow characteristics, caused by the combined action of the centrifugal, Coriolis and buoyancy forces.

Generally, the cross-sections considered are limited to the usual cases of circular, elliptical, and rectangular shapes with curved tubes of constants radius of curvature. More recently, some works considered trapezoidal cross-sections [7] or semicircular ones [28]. By using a perturbation method valid for low curvature ratio, Nourouzi and Biglari [11] in 2013 solved the Dean flow analytically in curved ducts with a rectangular cross-section.

It is proposed in this work to extend the availability of asymptotic analytical solutions to new ranges of cross-sectional shapes while considering fully developed steady state flows at low Dean numbers. The zero-order azimuthal velocity profiles for various cross-sections are given by exact analytical solutions obtained using the results of Lekner [29] and Letelier [30] in the case of straight tubes. Solutions for the nonhomogeneous biharmonic equation for the secondary flows are obtained 
by using Michel's general solution in polar coordinate for the homogeneous part and exact expressions for the particular solutions. Furthermore, the Fourier series decomposition of the solution is adopted to determine the values of the integration constants that allow satisfying the nonslip boundary conditions at the tube wall.

The original proposed method is validated by comparing the results with those of Dean in the case of a torus with a circular cross-section and then with those of Cumming [17] in the case of the elliptical cross-section. The characteristics introduced by the forms of non-circular cross-sections are highlighted by presenting the impact of the shape of the section on the spatial distribution and the intensity of the vortex's velocities.

In this context, this paper is organized as follows. The first part of section 2 presents the studied geometry; the second part gives the governing equations and the boundary conditions for the problem in dimensionless forms. In section 3 the analytical solution of the nonhomogeneous biharmonic equation for the secondary flows in tubes with a various cross-section where the axis of the tube is an arbitrary plane curve is described in detail together with the impact of the variation of the tube curvature on the flow. The results obtained for the velocity distribution and the Dean's vortexes for different cross-sections are presented in sections 3 and 4, where the impact of the crosssection flow is analysed. In addition, the first-order effects of the vortexes on the azimuthal velocity are given in section 5 . Finally, section 6 summarizes and discusses the obtained results.

\section{Mathematical Formulation}

\subsection{Coordinate System}

The steady flow of an incompressible Newtonian fluid in a curved pipe with a variable radius of curvature and an arbitrary cross-section is studied in a fixed orthogonal coordinate system $R(O, \vec{x}, \vec{y}, \vec{z})$. The axis of the tube is a plane curve $(\mathcal{C})$ of arbitrary equation $y=f(x)$ and $(x, y, z)$ are the cartesian coordinates. $R_{F}\left(O_{1}, \vec{\tau}, \vec{N}, \vec{B}\right)$ is a coordinate system where $O_{1}$ is located on $(\mathcal{C})$ and $(\vec{\tau}, \vec{N}, \vec{B})$ is the orthonormal triad with $\vec{\tau}$ the tangent vector to $(\mathcal{C}), \vec{N}$ the normal vector and $\vec{B}$ the binormal vector. $(s, r, \theta)$ is the curvilinear coordinate system with $s$ is the azimuthal coordinate along the plane curve $(\mathcal{C})$ and $(r, \theta)$ are polar coordinates in the plane $(\vec{N}, \vec{B}) \cdot\left(\overrightarrow{e_{s}}, \overrightarrow{e_{r}}, \overrightarrow{e_{\theta}}\right)$ are the associate unit vectors and $(X, Y)$ are the cartesian coordinates in the plane $(\vec{N}, \vec{B})$. It should be noted that when the radius of curvature is constant $\left(R_{c}=c s t\right)$, these coordinates coincide with the toroidal coordinates (Figure 1).

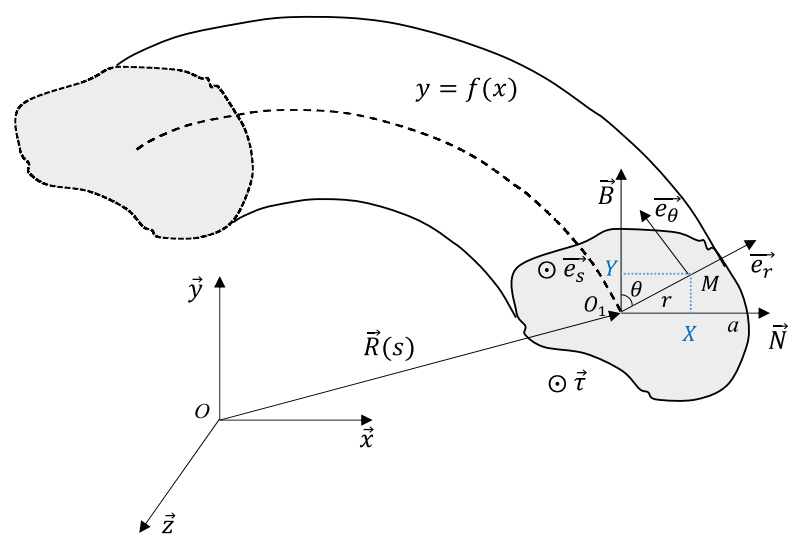

Fig. 1. Geometry of curved tube with variable cross-sectional shape 
Using these orthogonal systems, the position vector $\overrightarrow{O M}$ in $R(O, \vec{x}, \vec{y}, \vec{z})$ is written as

$\overrightarrow{O M}=\vec{R}(s)-r \sin (\theta) \vec{N}+r \cos (\theta) \vec{B} \quad ; \quad \theta>0$

where, $\vec{R}(s)=\overrightarrow{O O_{1}}$. The arc length element takes the form

$d \overrightarrow{\mathrm{OM}} \cdot d \overrightarrow{\mathrm{OM}}=h_{1}^{2}(s, r, \theta) d s^{2}+h_{2}^{2}(s, r, \theta) d r^{2}+h_{3}^{2}(s, r, \theta) d \theta^{2}$

where the metrics $h_{1}, h_{2}$ and $h_{3}$ for this system are given by

$h_{1}(s, r, \theta)=\left(1+\frac{r}{R_{c}(s)} \sin \theta\right), h_{2}(s, r, \theta)=1, h_{3}(s, r, \theta)=r$

where $R_{c}(s)=1 / k(s)$ is the radius of curvature and $k(s)$ is the curvature.

\subsection{Governing Equations}

Continuity equation

$\frac{\partial}{\partial s}(r u)+\frac{\partial}{\partial r}\left(h_{1} r v\right)+\frac{\partial}{\partial \theta}\left(h_{1} w\right)=0$

Azimuthal momentum equation $\left(\overrightarrow{e_{s}}\right)$

$\left(\frac{u}{h_{1}} \frac{\partial u}{\partial s}+\mathrm{v} \frac{\partial u}{\partial r}+\frac{w}{r} \frac{\partial u}{\partial \theta}\right)+\frac{u}{R_{c}(s)} \frac{(\mathrm{v} \sin \theta+w \cos \theta)}{h_{1}}=-\frac{1}{\rho h_{1}} \frac{\partial p}{\partial s}+v\left[\frac{1}{h_{1}} \frac{\partial}{\partial s}\left(\frac{1}{h_{1}} \frac{\partial u}{\partial s}\right)+\frac{1}{r} \frac{\partial}{\partial r}\left(\frac{r}{h_{1}} \frac{\partial\left(h_{1} u\right)}{\partial r}\right)+\right.$ $\left.\frac{1}{r^{2}} \frac{\partial}{\partial \theta}\left(\frac{1}{h_{1}} \frac{\partial\left(h_{1} u\right)}{\partial \theta}\right)+\frac{2}{h_{1}^{2}}\left\{\frac{\partial h_{1}}{\partial r} \frac{\partial \mathrm{v}}{\partial s}+\frac{1}{r} \frac{\partial h_{1}}{\partial \theta} \frac{\partial w}{\partial s}\right\}+\frac{1}{h_{1}}\left\{\mathrm{v} \frac{\partial}{\partial s}\left(\frac{1}{h_{1}} \frac{\partial h_{1}}{\partial r}\right)+\frac{w}{r} \frac{\partial}{\partial s}\left(\frac{1}{h_{1}} \frac{\partial h_{1}}{\partial \theta}\right)\right\}\right]$

Radial momentum equation $\left(\overrightarrow{\mathrm{e}_{\mathrm{r}}}\right)$

$\left(\frac{u}{h_{1}} \frac{\partial \mathrm{v}}{\partial s}+\mathrm{v} \frac{\partial \mathrm{v}}{\partial r}+\frac{w}{r} \frac{\partial \mathrm{v}}{\partial \theta}\right)-\frac{w^{2}}{r}-\frac{u^{2}}{R_{c}(s)} \frac{\sin \theta}{h_{1}}=-\frac{1}{\rho} \frac{\partial p}{\partial r}+v\left[\frac{1}{h_{1}} \frac{\partial}{\partial s}\left(\frac{1}{h_{1}} \frac{\partial \mathrm{v}}{\partial s}\right)+\frac{\partial^{2} \mathrm{v}}{\partial r^{2}}-\frac{\mathrm{v}}{r^{2}}+\frac{1}{r} \frac{\partial \mathrm{v}}{\partial r}+\frac{1}{r^{2}} \frac{\partial^{2} \mathrm{v}}{\partial \theta^{2}}-\frac{2}{r^{2}} \frac{\partial w}{\partial \theta}-\right.$

$\left.\frac{2}{h_{1}^{2}} \frac{\partial h_{1}}{\partial r} \frac{\partial u}{\partial s}+\mathrm{v} \frac{\partial}{\partial r}\left(\frac{1}{h_{1}} \frac{\partial h_{1}}{\partial r}\right)+\frac{w}{r} \frac{\partial}{\partial r}\left(\frac{1}{h_{1}} \frac{\partial h_{1}}{\partial \theta}\right)-\frac{u}{h_{1}} \frac{\partial}{\partial s}\left(\frac{1}{h_{1}} \frac{\partial h_{1}}{\partial r}\right)+\frac{1}{h_{1}}\left\{\frac{\partial h_{1}}{\partial r} \frac{\partial \mathrm{v}}{\partial r}+\frac{1}{r^{2}} \frac{\partial h_{1}}{\partial \theta} \frac{\partial \mathrm{v}}{\partial \theta}-\frac{2 w}{r^{2}} \frac{\partial h_{1}}{\partial \theta}\right\}\right]$

Tangential momentum equation $\left(\overrightarrow{e_{\theta}}\right)$

$\left(\frac{u}{h_{1}} \frac{\partial w}{\partial s}+\mathrm{v} \frac{\partial w}{\partial r}+\frac{w}{r} \frac{\partial w}{\partial \theta}\right)+\frac{\mathrm{v} w}{r}-\frac{u^{2}}{R_{c}(s)} \frac{\cos \theta}{h_{1}}=-\frac{1}{\rho r} \frac{\partial p}{\partial \theta}+v\left[\frac{1}{h_{1}} \frac{\partial}{\partial s}\left(\frac{1}{h_{1}} \frac{\partial w}{\partial s}\right)+\frac{\partial^{2} w}{\partial r^{2}}-\frac{w}{r^{2}}+\frac{1}{r} \frac{\partial w}{\partial r}+\frac{1}{r^{2}} \frac{\partial^{2} w}{\partial \theta^{2}}+\right.$
$\left.\frac{2}{r^{2}} \frac{\partial \mathrm{v}}{\partial \theta}-\frac{2}{h_{1}^{2} r} \frac{\partial h_{1}}{\partial \theta} \frac{\partial u}{\partial s}+\frac{\mathrm{v}}{r} \frac{\partial}{\partial \theta}\left(\frac{1}{h_{1}} \frac{\partial h_{1}}{\partial r}\right)+\frac{w}{r^{2}} \frac{\partial}{\partial \theta}\left(\frac{1}{h_{1}} \frac{\partial h_{1}}{\partial \theta}\right)-\frac{u}{r h_{1}} \frac{\partial}{\partial s}\left(\frac{1}{h_{1}} \frac{\partial h_{1}}{\partial \theta}\right)+\frac{1}{h_{1}}\left\{\frac{\partial h_{1}}{\partial r} \frac{\partial w}{\partial r}+\frac{1}{r^{2}} \frac{\partial h_{1}}{\partial \theta} \frac{\partial w}{\partial \theta}+\frac{w}{r} \frac{\partial h_{1}}{\partial r}\right\}\right]$

The dimensionless variables are defined as

$u=U u^{*}, \mathrm{v}=\frac{v}{a} \mathrm{v}^{*}, w=\frac{v}{a} w^{*}, p=p_{0} p^{*}, s=R_{m} s^{*}, r=a r^{*}, R_{c}=R_{m} R_{c}^{*}$

where $(\mathrm{u}, \mathrm{v}, \mathrm{w})$ are the velocity components along $\overrightarrow{e_{s}}, \overrightarrow{e_{r}}$ and $\overrightarrow{e_{\theta}}, U$ is a reference velocity, $v$ is the kinematic viscosity, $p$ is the pressure, $p_{0}$ is a reference pressure, $R_{m}$ and $a$ are respectively 
characteristic lengths related to the radius of curvature of the tube and to the hydraulic radius of the cross-section.

Assuming the ratio $\varepsilon=\frac{a}{R_{m}} \ll 1$, conservation equations for mass and momentum are reduced to the following dimensionless forms

Continuity Equation

$\frac{\partial\left(r^{*} v^{*}\right)}{\partial r^{*}}+\frac{\partial w^{*}}{\partial \theta}=0$

Azimuthal momentum equation $\left(\overrightarrow{\mathrm{e}_{\mathrm{s}}}\right)$

$\mathrm{v}^{*} \frac{\partial u^{*}}{\partial r^{*}}+\frac{w^{*}}{r^{*}} \frac{\partial u^{*}}{\partial \theta}=-\left\{\frac{p_{0} a \varepsilon}{\rho v U}\right\} \frac{\partial p^{*}}{\partial s^{*}}+\frac{\partial^{2} u^{*}}{\partial r^{* 2}}+\frac{1}{r^{*}} \frac{\partial u^{*}}{\partial r^{*}}+\frac{1}{r^{* 2}} \frac{\partial^{2} u^{*}}{\partial \theta^{2}}$

Radial momentum equation $\left(\overrightarrow{\mathrm{e}_{\mathrm{r}}}\right)$

$\mathrm{v}^{*} \frac{\partial \mathrm{v}^{*}}{\partial r^{*}}+\frac{w^{*}}{r^{*}} \frac{\partial \mathrm{v}^{*}}{\partial \theta}-\frac{w^{* 2}}{r^{*}}-\left\{2 R e^{2} \varepsilon\right\} \frac{u^{* 2} \sin \theta}{2 R_{c}^{*}\left(s^{*}\right)}=-\left\{\frac{p_{0} a^{2}}{\rho v^{2}}\right\} \frac{\partial p^{*}}{\partial r^{*}}-\frac{1}{r^{*}} \frac{\partial}{\partial \theta}\left(\frac{\partial w^{*}}{\partial r^{*}}+\frac{w^{*}}{r^{*}}-\frac{1}{r^{*}} \frac{\partial \mathrm{v}^{*}}{\partial \theta}\right)$

Tangential momentum equation $\left(\overrightarrow{\mathrm{e}_{\theta}}\right)$

$\mathrm{V}^{*} \frac{\partial w^{*}}{\partial r^{*}}+\frac{w^{*}}{r^{*}} \frac{\partial w^{*}}{\partial \theta}+\frac{\mathrm{v}^{*} w^{*}}{r^{*}}-\left\{2 R e^{2} \varepsilon\right\} \frac{u^{* 2} \cos \theta}{2 R_{c}^{*}\left(s^{*}\right)}=-\left\{\frac{p_{0} a^{2}}{\rho v^{2}}\right\} \frac{1}{r^{*}} \frac{\partial p^{*}}{\partial \theta}+\frac{\partial}{\partial r^{*}}\left(\frac{\partial w^{*}}{\partial r^{*}}+\frac{w^{*}}{r^{*}}-\frac{1}{r^{*}} \frac{\partial \mathrm{v}^{*}}{\partial \theta}\right)$

where $\left(u^{*}, \mathrm{v}^{*}, w^{*}\right)$ are the non-dimensional velocity components along $\overrightarrow{e_{s}}, \overrightarrow{e_{r}}$ and $\overrightarrow{e_{\theta}}, \rho$ is the fluid density, $R e=\frac{U a}{v}$ is the Reynolds number and $D=2 R e^{2} \varepsilon$ the dimensionless number defined by Dean [13]. The no-slip boundary conditions are given by $u^{*}=\mathrm{v}^{*}=w^{*}=0$ at the tube wall.

In the case of a constant radius of curvature and for circular cross-section, the tube is a torus, and the system of Eq. (9)-(12) is identical to that used by Dean in the toroidal coordinate system. While, if $R_{c}^{*} \rightarrow \infty$, one obtains the equations on the system of the cylindrical coordinates for a straight tube.

\section{Resolution}

For low values of the number $D$, solutions of Eq. (9)-(12) with the associated boundary conditions are sought in the form asymptotic expansions in $D$ written as

$$
\begin{aligned}
& u^{*}=u_{0}^{*}\left(s^{*}, r^{*}, \theta\right)+D u_{1}^{*}\left(s^{*}, r^{*}, \theta\right)+D^{2} u_{2}^{*}\left(s^{*}, r^{*}, \theta\right)+\cdots \\
& \mathrm{v}^{*}=D \mathrm{v}_{1}^{*}\left(s^{*}, r^{*}, \theta\right)+D^{2} \mathrm{v}_{2}^{*}\left(s^{*}, r^{*}, \theta\right)+\cdots \\
& w^{*}=D w_{1}^{*}\left(s^{*}, r^{*}, \theta\right)+D^{2} w_{2}^{*}\left(s^{*}, r^{*}, \theta\right)+\cdots \\
& p^{*}=p_{0}^{*}\left(s^{*}\right)+D p_{1}^{*}\left(s^{*}, r^{*}, \theta\right)+D^{2} p_{2}^{*}\left(s^{*}, r^{*}, \theta\right)+\cdots
\end{aligned}
$$

where index 0, 1 and 2 represent respectively the variables at zero, first and second order in $D$. 
At zero order in number $D$, Eq. (9)-(12) are reduced to

$$
\left\{\begin{array}{l}
0=-\frac{\partial p_{0}^{*}}{\partial s^{*}}+\frac{\partial^{2} u_{0}^{*}}{\partial r^{*}}+\frac{1}{r^{*}} \frac{\partial u_{0}^{*}}{\partial r^{*}}+\frac{1}{r^{*}} \frac{\partial^{2} u_{0}^{*}}{\partial \theta^{2}} \\
0=-\frac{\partial p_{0}^{*}}{\partial r^{*}} \\
0=-\frac{1}{r^{*}} \frac{\partial p_{0}^{*}}{\partial \theta}
\end{array}\right.
$$

The zero-order boundary conditions set vanishing values of the velocity $u_{0}^{*}$ at the wall. It should be noted at this stage that the shape of the section is not yet defined, and therefore these boundary conditions are not explicit.

For the system of Eq. (14), the classical analytical solutions are those obtained in the case of a circular section with a parabolic velocity profile, then that generally presented in the case of triangular and elliptical cross-section conduits, as well as the developments obtained for the rectangular cross-sections and for a reduced number of other cross-section shapes [31]. Based on the method presented by Lekner [29] and by Letelier [30], let us note that the azimuthal pressure gradient at zero-order depends only on the variable $s$, it could, therefore, be considered a solution $u_{0}^{*}$ in the form

$u_{0}^{*}\left(s^{*}, r^{*}, \theta\right)=\left(-\frac{1}{4} \frac{\partial p_{0}^{*}}{\partial s^{*}}\right)\left[1-r^{* 2}+h\left(r^{*}, \theta\right)\right]$

where $h\left(r^{*}, \theta\right)$ is any function satisfying the polar harmonic equation. By retaining for $h\left(r^{*}, \theta\right)$ only the functions that cancel $u_{0}^{*}$ on a closed contour, we see that a wide range of functions remain available, including the real and imaginary parts of many analytical functions, some examples of which are given by Lekner [29] with $h\left(r^{*}, \theta\right)=\left(X^{*}+i Y^{*}\right)^{n}$ with $n \in \mathbb{N}, h\left(r^{*}, \theta\right)=\sin \left(X^{*}+i Y^{*}\right)$ and $h\left(r^{*}, \theta\right)=e^{i\left(X^{*}+i Y^{*}\right)}$, where $\left(X^{*}, Y^{*}\right)$ are the dimensionless cartesian coordinates. On the other hand, note that when $h\left(r^{*}, \theta\right)=0$, the section is circular.

In what follows, we will consider $h\left(r^{*}, \theta\right)=r^{* \gamma} \sin (\gamma \theta)$ which corresponds to the real part of the analytical function $Z^{* \gamma}=\left(X^{*}+i Y^{*}\right)^{\gamma}=r^{* \gamma} e^{\gamma i \theta}$ where $\gamma$ is a positive constant, $X^{*}=r^{*} \sin \theta$ and $Y^{*}=r^{*} \cos \theta$. The velocity profile, in polar coordinate, based on the real part of the function $Z^{* \gamma}$ can be written as [29]

$u_{0}^{*}\left(s^{*}, r^{*}, \theta\right)=\left(-\frac{1}{4} \frac{\partial p_{0}^{*}}{\partial s^{*}}\right)\left[1-r^{* 2}+\delta r^{* \gamma} \sin (\gamma \theta)\right]$

here, $\delta$ denotes a correction parameter and $r^{*}$ is defined as $r^{*}=\sqrt{X^{* 2}+Y^{* 2}}$. We note that at the limits $\gamma \rightarrow 0$ and $\gamma \rightarrow \infty$ we recover the circular form. The implicit polar equation for $R^{*}(\theta)$ obtained by setting a vanishing velocity on the tube wall is given by

$1-R^{* 2}(\theta)+\delta R^{* \gamma}(\theta) \sin (\gamma \theta)=0$

Figure 2(a)-(c) shows a variety of cross-sectional pipe shapes obtained for different values of the parameters $(\delta, \gamma)$ in Eq. (17). 


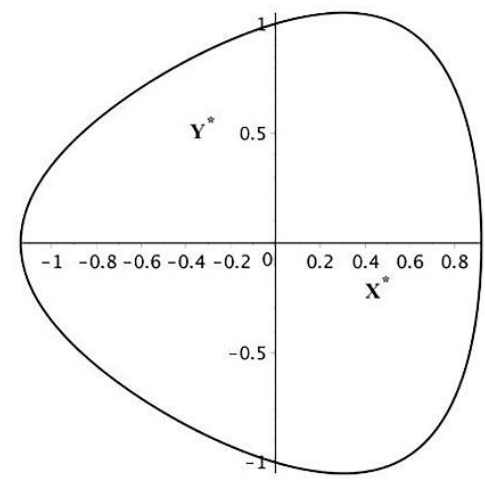

(a)

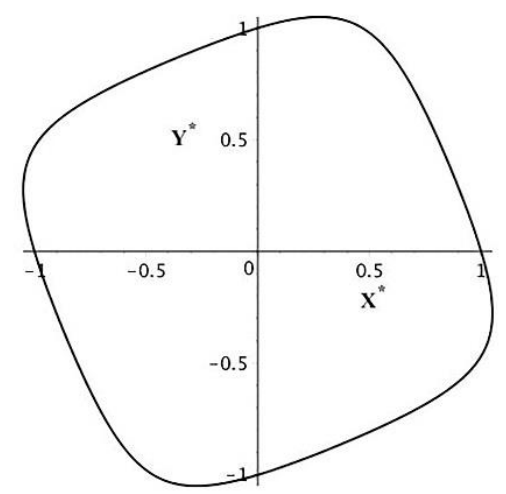

(b)

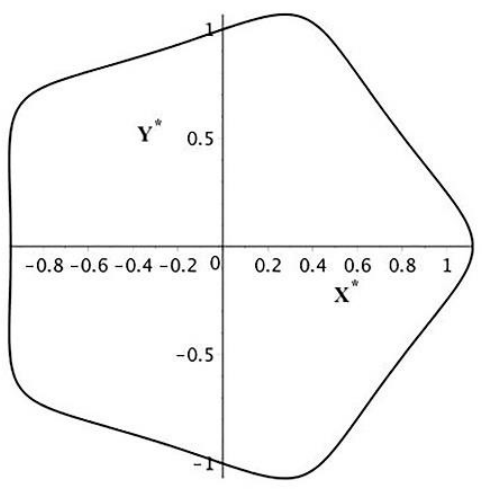

(c)

Fig. 2. The variety of pipe cross-sectional shapes for different values of $\delta$ and $\gamma(\mathrm{a}): \gamma=3, \delta=0.2$, (b): $\gamma=4, \delta=0.15$, (c): $\gamma=5, \delta=0.15$

At first order in number $D$, the system in Eq. (9)-(12) reduces to

$\frac{\partial\left(r^{*} v_{1}^{*}\right)}{\partial r^{*}}+\frac{\partial w_{1}^{*}}{\partial \theta}=0$

$\mathrm{V}_{1}^{*} \frac{\partial u_{0}^{*}}{\partial r^{*}}+\frac{w_{1}^{*}}{r^{*}} \frac{\partial u_{0}^{*}}{\partial \theta}=-\left\{\frac{p_{0} a \varepsilon}{\rho v U}\right\} \frac{\partial p_{1}^{*}}{\partial s^{*}}+\frac{\partial^{2} u_{1}^{*}}{\partial r^{2}}+\frac{1}{r^{*}} \frac{\partial u_{1}^{*}}{\partial r^{*}}+\frac{1}{r^{*}} \frac{\partial^{2} u_{1}^{*}}{\partial \theta^{2}}$

$-\frac{u_{0}^{* 2} \sin \theta}{2 R_{c}^{*}\left(s^{*}\right)}=-\left\{\frac{p_{0} a^{2}}{\rho v^{2}}\right\} \frac{\partial p_{1}^{*}}{\partial r^{*}}-\frac{1}{r^{*}} \frac{\partial}{\partial \theta}\left(\frac{\partial w_{1}^{*}}{\partial r^{*}}+\frac{w_{1}^{*}}{r^{*}}-\frac{1}{r^{*}} \frac{\partial \mathrm{v}_{1}^{*}}{\partial \theta}\right)$

$-\frac{u_{0}^{* 2} \cos \theta}{2 R_{c}^{*}\left(s^{*}\right)}=-\left\{\frac{p_{0} a^{2}}{\rho v^{2}}\right\} \frac{1}{r^{*}} \frac{\partial p_{1}^{*}}{\partial \theta}+\frac{\partial}{\partial r^{*}}\left(\frac{\partial w_{1}^{*}}{\partial r^{*}}+\frac{w_{1}^{*}}{r^{*}}-\frac{1}{r^{*}} \frac{\partial \mathrm{v}_{1}^{*}}{\partial \theta}\right)$

Eq. (18) implies the existence of a dimensionless pseudo-stream function $\psi_{1}^{*}$ defined by

$\mathrm{v}_{1}^{*}=-\frac{1}{r^{*}} \frac{\partial \psi_{1}^{*}}{\partial \theta}, w_{1}^{*}=\frac{\partial \psi_{1}^{*}}{\partial r^{*}}$

Inserting the expression in Eq. (22) in the system of Eq. (19)-(21) and eliminating the pressure terms between Eq. (20) and Eq. (21), give the following equations

$\frac{1}{r^{*}}\left(-\frac{\partial \psi_{1}^{*}}{\partial \theta} \frac{\partial u_{0}^{*}}{\partial r^{*}}+\frac{\partial \psi_{1}^{*}}{\partial r^{*}} \frac{\partial u_{0}^{*}}{\partial \theta}\right)=-\left\{\frac{p_{0} a \varepsilon}{\rho v U}\right\} \frac{\partial p_{1}^{*}}{\partial s^{*}}+\frac{\partial^{2} u_{1}^{*}}{\partial r^{* 2}}+\frac{1}{r^{*}} \frac{\partial u_{1}^{*}}{\partial r^{*}}+\frac{1}{r^{* 2}} \frac{\partial^{2} u_{1}^{*}}{\partial \theta^{2}}$

$\frac{1}{r^{*}}\left(-\frac{\partial \psi_{1}^{*}}{\partial \theta} \frac{\partial}{\partial r^{*}}+\frac{\partial \psi_{1}^{*}}{\partial r^{*}} \frac{\partial}{\partial \theta}\right) \Delta \psi_{1}^{*}+\frac{D u_{0}^{*}}{R_{C}^{*}\left(s^{*}\right)}\left(-\frac{\partial u_{0}^{*}}{\partial r^{*}} \cos \theta+\frac{\partial u_{0}^{*}}{\partial \theta} \frac{\sin \theta}{r^{*}}\right)=\Delta\left(\Delta \psi_{1}^{*}\right)$

Replacing the expression in Eq. (16) in Eq. (24), the equation for $\psi_{1}^{*}$ can be written as

$$
\begin{aligned}
& \Delta\left(\Delta \psi_{1}^{*}\right)=\frac{1}{R_{c}^{*}\left(s^{*}\right)}\left(-\frac{1}{4}\left(\frac{\partial p_{0}^{*}}{\partial s^{*}}\right)\right)^{2}\left[\left(2\left(r^{*}-r^{* 3}\right)+\frac{\gamma \delta^{2}}{2}\right) \cos (\theta)+\delta\left(\gamma\left(1-r^{* 2}\right) r^{* \gamma-1}+\right.\right. \\
& \left.\left.r^{* \gamma+1}\right) \sin ((\gamma-1) \theta)+\delta r^{* \gamma+1} \sin ((\gamma+1) \theta)-\frac{\delta^{2} \gamma}{2} r^{* 2 \gamma-1} \cos ((2 \gamma-1) \theta)\right]
\end{aligned}
$$


with the no-slip boundary conditions $\left.\frac{\partial \psi_{1}^{*}}{\partial r^{*}}\right|_{r^{*}=R^{*}(\theta)}=0$ and $\left.\frac{\partial \psi_{1}^{*}}{\partial \theta}\right|_{r^{*}=R^{*}(\theta)}=0$ at the tube wall, with $R^{*}(\theta)$ is the dimensionless polar equation of the tube wall .

By analytically calculating the particular solutions of Eq. (25) and taking into account Michel's solution [32] for the biharmonic equations in polar coordinates with periodicity condition to establish the solution of the homogeneous equation, we get the following form for the solution of Eq. (25)

$$
\begin{aligned}
& \psi_{1}^{*}\left(s^{*}, r^{*}, \theta\right)=\frac{1}{R_{c}^{*}\left(s^{*}\right)}\left(-\frac{1}{4}\left(\frac{\partial p_{0}^{*}}{\partial s^{*}}\right)\right)^{2}\left(\frac { 1 } { 3 2 } \left[\left(\frac{r^{* 5}}{3}-\frac{r^{* 7}}{18}-\frac{\delta^{2} r^{* 2 \gamma+3}}{\left(\gamma^{2}+3 \gamma+2\right)(\gamma+1)}\right) \cos (\theta)+\delta\left(\frac{(1+\gamma) r^{* \gamma+5}}{3(\gamma+1)(\gamma+2)}-\right.\right.\right. \\
& \left.\left.\frac{r^{* \gamma+3}}{(\gamma+1)}\right) \sin ((\gamma-1) \theta)+\delta \frac{r^{* \gamma+5}}{(\gamma+3)(\gamma+2)} \sin ((\gamma+1) \theta)+\frac{\delta^{2}}{4(2 \gamma+1)} r^{* 2 \gamma+3} \cos ((2 \gamma-1) \theta)\right]+ \\
& \left.\sum_{n=1}^{K}\left\{\left(A_{n} r^{* n}+B_{n} r^{* n+2}\right) \cos (n \theta)+\left(C_{n} r^{* n}+D_{n} r^{* n+2}\right) \sin (n \theta)\right\}\right)
\end{aligned}
$$

where $A_{n}, B_{n}, C_{n}, D_{n}$ are unknown constants to be determined for $n=1 . K(K \in \mathbb{N})$. A priori, $K$ is infinite, but the sine and cosine series in Eq. (26) will be truncated to a given order, which depends on the considered cross-sectional shapes as well as on the precision sought.

The difficulty in solving a non-homogeneous biharmonic equation lies in determining integration constants that allow the solution to satisfy the no-slip conditions at the wall. First, let us note that, by using Eq. (26), the no-slip conditions setting $\psi_{1}^{*}\left(r^{*}=R^{*}(\theta), \theta\right)=0$ and $\left.\frac{\partial \psi_{1}^{*}\left(r^{*}, \theta\right)}{\partial r^{*}}\right|_{r^{*}=R^{*}(\theta)}=0$ are given by

$$
\begin{aligned}
& \frac{1}{32}\left[\left(\frac{R^{* 5}(\theta)}{3}-\frac{R^{* 7}(\theta)}{18}+\frac{\delta^{2} R^{* 2 \gamma+3}(\theta)}{\left(\gamma^{2}+3 \gamma+2\right)(\gamma+1)}\right) \cos (\theta)+\delta\left(\frac{(1-\gamma) R^{* \gamma+5}(\theta)}{3(\gamma+1)(\gamma+2)}+\frac{R^{* \gamma+3}(\theta)}{(\gamma+1)}\right) \sin ((\gamma-1) \theta)+\right. \\
& \left.\delta \frac{R^{* \gamma+5}(\theta)}{(\gamma+3)(\gamma+2)} \sin ((\gamma+1) \theta)-\frac{\delta^{2}}{4(2 \gamma+1)} R^{* 2 \gamma+3}(\theta) \cos ((2 \gamma-1) \theta)\right]+\sum_{n=1}^{K}\left\{\left(A_{n} R^{* n}(\theta)+\right.\right. \\
& \left.\left.B_{n} R^{* n+2}(\theta)\right) \cos (n \theta)+\left(C_{n} R^{* n}(\theta)+D_{n} R^{* n+2}(\theta)\right) \sin (n \theta)\right\}=0 \\
& \frac{1}{32}\left[\left(\frac{5 R^{* 4}(\theta)}{3}-\frac{7 R^{* 6}(\theta)}{18}+\frac{\delta^{2}(2 \gamma+3) R^{* 2 \gamma+2}(\theta)}{\left(\gamma^{2}+3 \gamma+2\right)(\gamma+1)}\right) \cos (\theta)+\delta\left(\frac{(1-\gamma)(\gamma+5) R^{* \gamma+4}(\theta)}{3(\gamma+1)(\gamma+2)}+\frac{(\gamma+3) R^{* \gamma+2}(\theta)}{(\gamma+1)}\right) \sin ((\gamma-\right. \\
& \text { 1) } \left.\theta)+\delta \frac{(\gamma+5) R^{* \gamma+4}(\theta)}{(\gamma+3)(\gamma+2)} \sin ((\gamma+1) \theta)-\frac{\delta^{2}(2 \gamma+3)}{4(2 \gamma+1)} R^{* 2 \gamma+2}(\theta) \cos ((2 \gamma-1) \theta)\right]+ \\
& \sum_{n=1}^{K}\left\{\left(n A_{n} R^{* n-1}(\theta)+(n+2) B_{n} R^{* n+1}(\theta)\right) \cos (n \theta)+\left(n C_{n} R^{* n-1}(\theta)+(n+\right.\right. \\
& \text { 2) } \left.\left.D_{n} R^{* n+1}(\theta)\right) \sin (n \theta)\right\}=0
\end{aligned}
$$

By taking into account the polar equation of the tube wall $r^{*}=R^{*}(\theta)$, and remarking, due again to the necessary periodicity in $\theta$ of the solution, that for all integer $m \in\{1, K\}$ the function $\psi_{1}^{*}$ must satisfy

$$
\begin{aligned}
& \int_{0}^{2 \pi} \psi_{1}^{*}\left(r^{*}=R^{*}(\theta), \theta\right) \cos (m \theta) d \theta=0 \\
& \int_{0}^{2 \pi} \psi_{1}^{*}\left(r^{*}=R^{*}(\theta), \theta\right) \sin (m \theta) d \theta=0 \\
& \int_{0}^{2 \pi} \frac{\partial \psi_{1}^{*}}{\partial r^{*}}\left(r^{*}=R^{*}(\theta), \theta\right) \cos (m \theta) d \theta=0 \\
& \int_{0}^{2 \pi} \frac{\partial \psi_{1}^{*}}{\partial r^{*}}\left(r^{*}=R^{*}(\theta), \theta\right) \sin (m \theta) d \theta=0
\end{aligned}
$$


One obtains a system of $4 K$ algebraic equations for the $4 K$ constants $A_{n}, B_{n}, C_{n}, D_{n}$ whose resolution, by using a symbolic mathematical software, allows to access to analytical expressions of these integration constants to a given order. Therefore, using Eq. (24), we have an estimate of $\psi_{1}^{*}$ at rank $K$ noted $\psi_{1}^{*(K)}$. This estimate exactly satisfies the Eq. (24) and fulfills the no-slip boundary conditions to a given degree of precision.

It should be noted that the integrals in Eq. (29)-(32) may require an extended calculation time, especially if we consider high $K$ values. Therefore, it is useful to consider methods that optimize the computation time, and the seventh order Newton-Cotes method is used for the results presented here. Also, to enhance the accuracy of calculations, the last term of rank $K+1$ has been added to the series in Eq. (24), and a closing rule imposing that this term of rank $K+1$ cancels exactly the projection on $\cos ((K+1) \theta)$ and $\sin ((K+1) \theta)$ of the estimate of $\psi_{1}^{*(K)}$ has been introduced.

Other methods can be used to solve Eq. (24) with the associated boundary conditions, including finite difference [33] or finite element methods [34], but which require the generation of appropriate meshes for each new geometry. Similarly, mesh-free methods based on radial fundamental solutions of the biharmonic equation can be used, such as the method of fundamental solutions (MFS) as in [35]. In our case, it has been preferred to take advantage of the availability of particular solutions given by exact analytical expressions and to introduce an original method to allow the satisfaction of the boundary conditions. By using the symbolic calculation possibilities, semi-analytical expressions are obtained and can be useful for optimization purposes.

The validation of the method is carried out by calculating the values of $\psi_{1}^{*(K)}$ and its derivatives at the boundary of the domain to ensure that the no-slip conditions are fulfilled, knowing that the form used to express $\psi_{1}^{*(K)}$ satisfies the Eq. (24) inside the domain. The obtained iso contours of the function $\psi_{1}^{*(K)}$ are presented in Figure 3 and compared favorably with those of Dean for the circular cross-section (Figure 3(a)) and Cumming for an elliptical cross-section (Figure 3(b)). In the latter case, the estimation of the accuracy of the solution was carried out by calculating the relative error $E_{r}=$ $\frac{\psi_{1}^{*}-\psi_{\text {Cumming }}^{*}}{\overline{\psi_{\text {Cumming }}^{*}}}$ between the Cumming solution $\psi_{\text {Cumming }}^{*}$ and that obtained by the present method $\psi_{1}^{*}$ (where $\overline{\psi_{\text {Cumming }}^{*}}$ is the average value of $\psi_{\text {Cumming }}^{*}$ ), this error is of order $10^{-7}$ as shown in Figure 4.

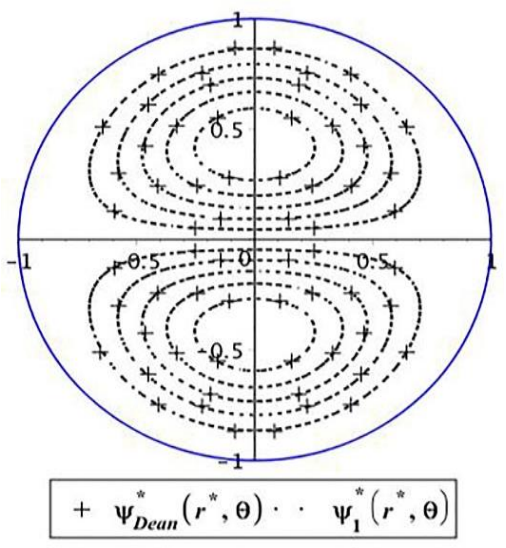

(a)

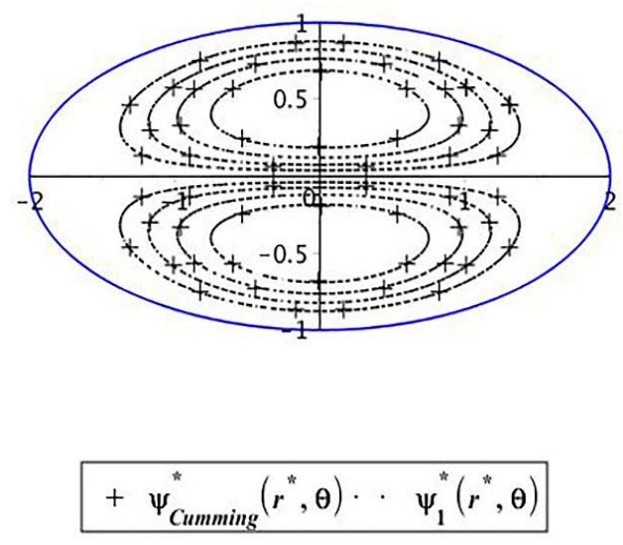

(b)

Fig. 3. The iso contours of the $\psi_{1}^{*(K)}$ function with constant $R_{c}^{*}\left(s^{*}\right)$ for: (a) Circular cross section, (b) Elliptical cross section 


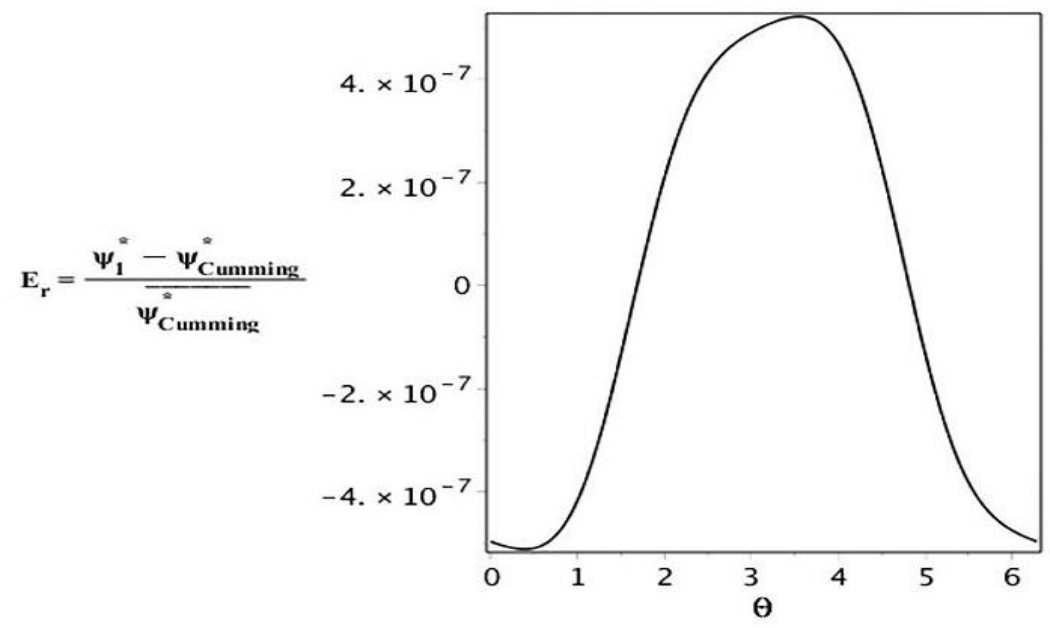

Fig. 4. The generated error between the Cumming solution

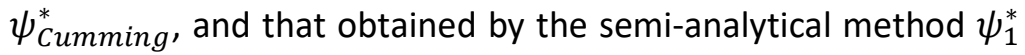
in terms of $\theta$ and for $r^{*}=0.1$

The results for other geometries are presented in Figure 5 by representing respectively the iso contours of the $\psi_{1}^{*}$ function and the velocity field $\overrightarrow{V^{*}}=\mathrm{v}_{1}^{*} \overrightarrow{e_{r}}+w_{1}^{*} \overrightarrow{e_{\theta}}$ in each case for fixed values of $R_{c}^{*}\left(s^{*}\right)$, these figures show that the variation of the cross-section shapes modifies the velocity distribution and the form of the Dean's vortexes.

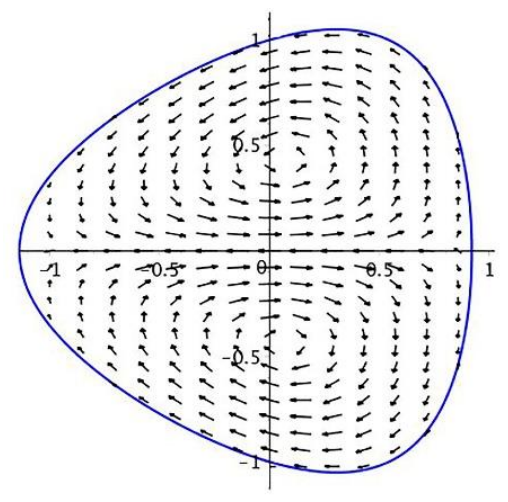

(a)

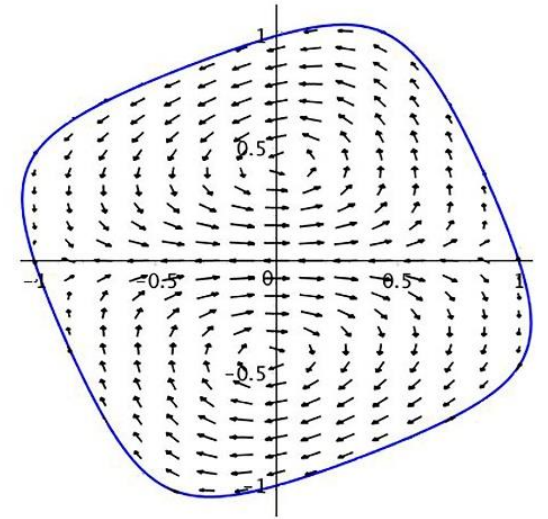

(b)

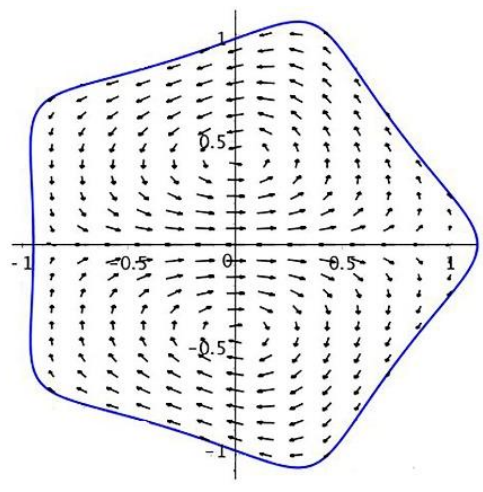

(c)

Fig. 5. The velocity field $\overrightarrow{V^{*}}$ in each section with $R_{c}^{*}\left(s^{*}\right)=c s t$ and for (a): $\gamma=3, \delta=0.2$, (b): $\gamma=4, \delta=$ $0.15,(\mathrm{c}): \gamma=5, \delta=0.15$

It should also be mentioned that the approach presented allows considering different form for the function $f\left(x^{*}\right)$. In the case of circular cross-sections, we present in Figure 6 the variation of the intensity of the maximum of the velocity on the axis defined by $V_{\max }^{*}\left(\mathrm{R}_{\mathrm{c}}^{*}\left(\mathrm{~s}^{*}\right), X^{*}=0, Y^{*}=0\right)=$ $\sqrt{\mathrm{v}_{1}^{* 2}+w_{1}^{* 2}}$ along the curved tube. We note that the variation in the radius of curvature results in a variation in the magnitude of the secondary flow velocities along the curved pipe. 


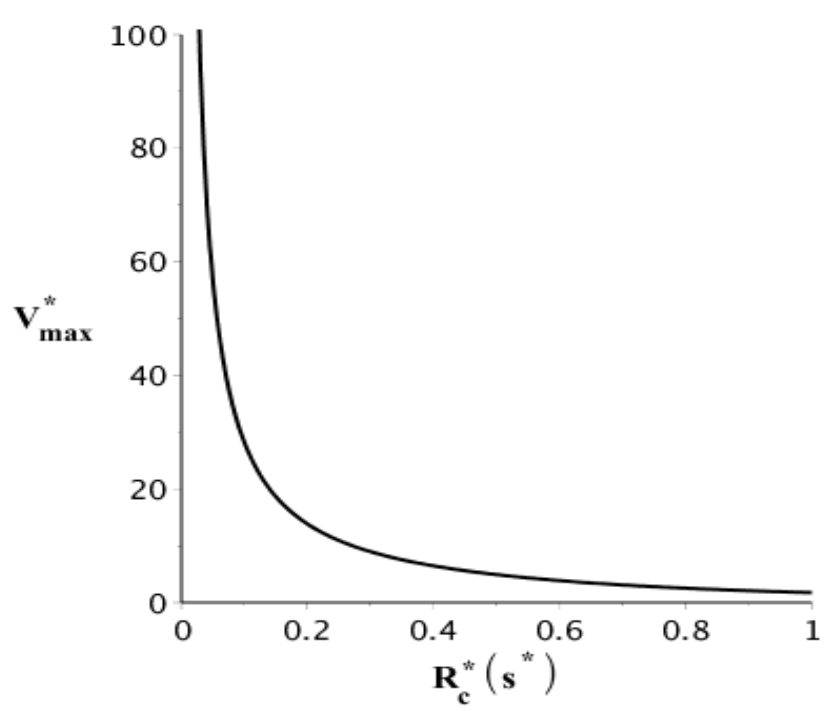

Fig. 6. The maximum of the velocity on the axis $V_{\text {max }}^{*}$ in terms of the $\mathrm{R}_{\mathrm{c}}^{*}\left(\mathrm{~s}^{*}\right)$

In addition, notice that the solutions presented here are obtained by solving equations identical to those of Dean, the only changes being the modifications of boundary conditions related to the new cross section's shapes introduced and the changes in the zero-order solution given by Eq. (16). We can therefore consider that these solutions for various cross-sections shapes have the same range of validity as that of Dean's solution for the case of a circular section. The validity of Dean's solution has been limited by Dean to values of $\operatorname{Re} \sqrt{\varepsilon} \leq 34$, and investigated experimentally by White (1929), Taylor 1929, and Adler 1934 (see Berger and Talbot [8]). However, more recently, Canton et al., [36] in 2017 presented a detailed investigation of the flow in a toroidal pipe. They showed in particular, from the analysis of more than 110000 numerical results, that the flow could not be analyzed starting from Dean number only but that it is necessary to consider two independent parameters separately, namely the Reynolds number and the pipe curvature. They further concluded that the validity should be limited to $\operatorname{Re} \sqrt{\varepsilon} \leq 10$ and only for very low values of curvature. For the present study, and insofar as the interest is mainly devoted to the effects of changes in cross section's shapes, we use Dean's approach while noting that the validity of the presented solutions is more limited than suggested by Dean.

\section{Impact of The Shape of The Section}

To illustrate the influence of the cross-section shape on the velocity distribution in the section, Figure 7 shows the intensity of the vortex velocity defined by the scalar field $V_{m}^{*}\left(r^{*}, \theta\right)=\sqrt{\mathrm{v}_{1}^{* 2}+w_{1}^{* 2}}$ in a cross-section. In the circular cross-section case, Figure 7(a), it is noted that the maximum of the vortex intensity $M_{0}$ lies on the axis $\theta=0$ and that there are two local maxima $M_{1}$ and $M_{2}$ located symmetrically relatively to the axis $\theta=0$. In the case of the other sections, there are other local maximums in the section corresponding to the points $M_{1}, M_{2}, M_{3}$ and $M_{4}$ in Figure 7(b)-(d).

Moreover, the shape of the cross-section will also modify the pressure-flow relationship. 


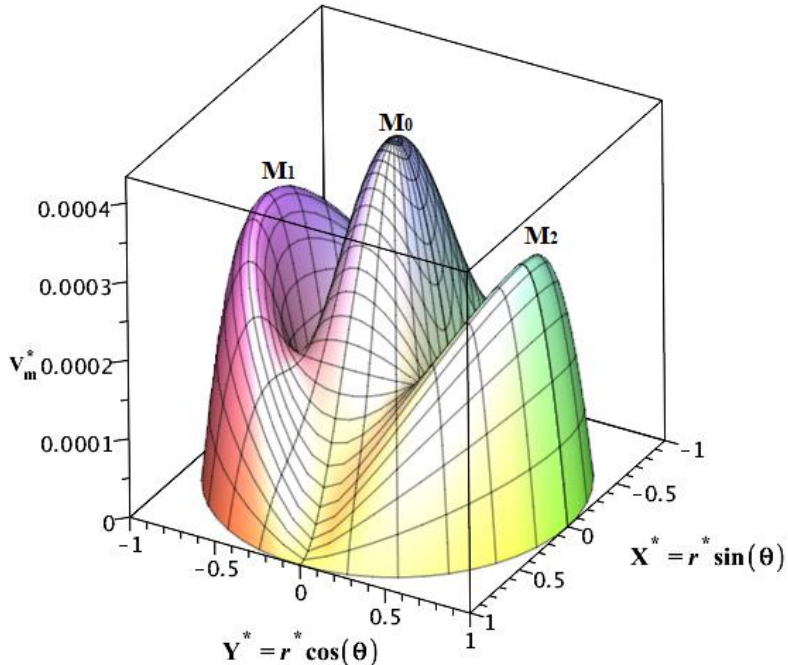

(a)

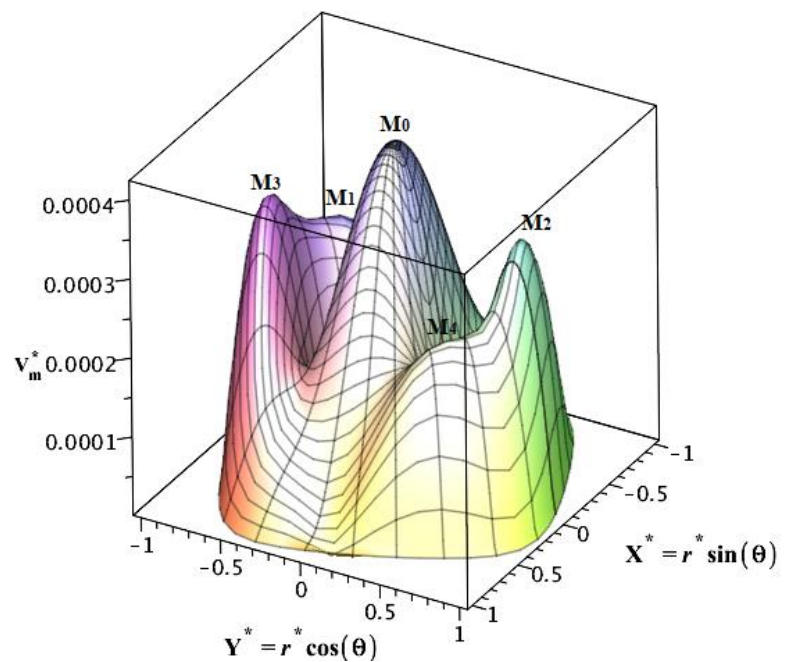

(c)

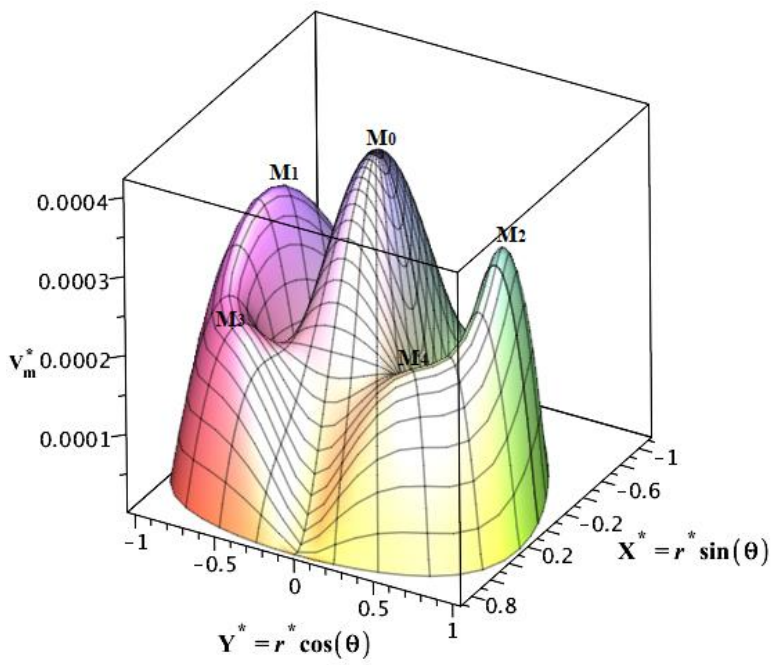

(b)

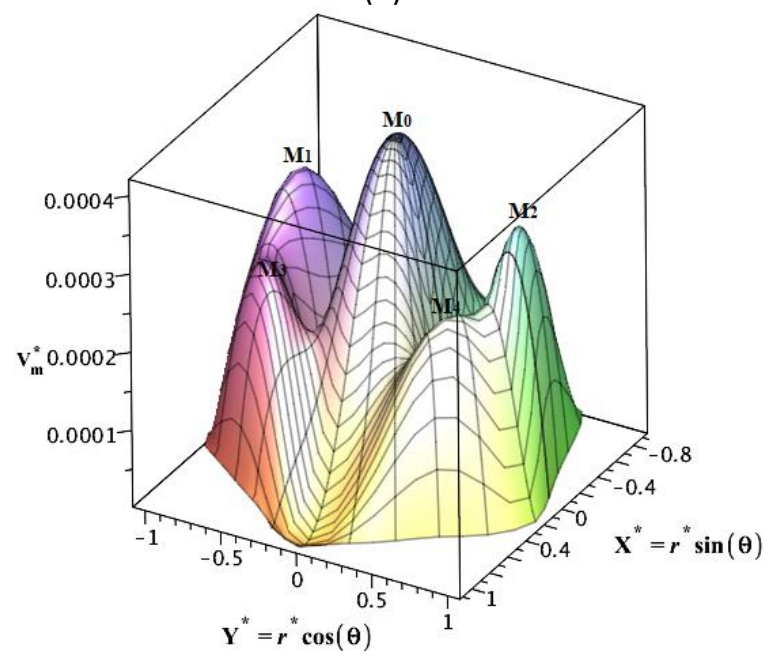

(d)

Fig. 7. The velocity distribution $V_{m}^{*}$ in $3 D$ for each section (a): $\gamma=0, \delta=0,(\mathrm{~b}): \gamma=3, \delta=0.2$, (c): $\gamma=$ $4, \delta=0.15,(\mathrm{~d}): \gamma=5, \delta=0.15$

\section{The First Order Azimuthal Velocity for Low $D$ Numbers and The Pressure-Flow Relationship}

In order to estimate the effects of tube curvature on azimuthal velocity of the flow, the first-order terms for azimuthal velocities are calculated using the same method as that of Dean [14], except that in our case the cross-section of the tube is no longer circular but is one of the shapes defined by the relation in Eq. (17).

So, at first order of $D$, the azimuthal Eq. (23) became

$\frac{1}{r^{*}}\left(-\frac{\partial \psi_{1}^{*}}{\partial \theta} \frac{\partial u_{0}^{*}}{\partial r^{*}}+\frac{\partial \psi_{1}^{*}}{\partial r^{*}} \frac{\partial u_{0}^{*}}{\partial \theta}\right)=\frac{\partial^{2} u_{1}^{*}}{\partial r^{* 2}}+\frac{1}{r^{*}} \frac{\partial u_{1}^{*}}{\partial r^{*}}+\frac{1}{r^{* 2}} \frac{\partial^{2} u_{1}^{*}}{\partial \theta^{2}}$

Replacing $\psi_{1}^{*}$ and $u_{0}^{*}$ by their previously obtained expressions, the solution of the Eq. (33) takes the following form 


$$
\begin{aligned}
& u_{1}^{*}\left(s^{*}, r^{*}, \theta\right)=\frac{1}{R_{c}^{*}\left(s^{*}\right)}\left(-\frac{1}{4}\left(\frac{\partial p_{0}^{*}}{\partial s^{*}}\right)\right)^{3}\left[\sum_{L=1}^{3} P_{L}^{1}\left(r^{*}\right) \cos \left(\lambda_{L}^{1} \theta\right)+\sum_{L=1}^{3} P_{L}^{2}\left(r^{*}\right) \sin \left(\lambda_{L}^{2} \theta\right)+\right. \\
& \sum_{n=1}^{K}\left\{P_{n}^{* 1}\left(r^{*}\right) \cos ((\gamma-n) \theta)+P_{n}^{* 2}\left(r^{*}\right) \sin ((\gamma-n) \theta)+P_{n}^{* * 1}\left(r^{*}\right) \cos ((\gamma+n) \theta)+\right. \\
& \left.P_{n}^{* * 2}\left(r^{*}\right) \sin ((\gamma+n) \theta)+P_{n}^{* * * 1}\left(r^{*}\right) \cos (n \theta)++P_{n}^{* * * 2}\left(r^{*}\right) \sin (n \theta)\right\}+\sum_{n=1}^{K_{1}}\left\{J_{1 n} r^{* n} \cos (n \theta)+\right. \\
& \left.\left.J_{2 n} r^{* n} \sin (n \theta)\right\}\right]
\end{aligned}
$$

where $P_{L}^{1}\left(r^{*}\right), P_{L}^{2}\left(r^{*}\right), P_{n}^{* 1}\left(r^{*}\right), P_{n}^{* * 1}\left(r^{*}\right), P_{n}^{* * * 1}\left(r^{*}\right), P_{n}^{* 2}\left(r^{*}\right), P_{n}^{* * 2}\left(r^{*}\right)$ and $P_{n}^{* * * 2}\left(r^{*}\right)$ are known polynomials whose expressions are given in the first order azimuthal velocity for low Dean numbers [33-34], the $\lambda_{L}$ are integers given by: $\lambda_{1}^{1}=(\gamma-1), \lambda_{2}^{1}=(\gamma+1), \lambda_{3}^{1}=(3 \gamma-1), \lambda_{1}^{2}=1, \lambda_{2}^{2}=$ $(2 \gamma-1), \lambda_{3}^{2}=(2 \gamma-1), J_{1 n}$ and $J_{2 n}$ are unknown constants to be determined for $n=1 . . K_{1}\left(K_{1} \in\right.$ $\mathbb{N}$ ), with $K_{1}$ a priori is infinite and also depends on the considered cross-sectional shapes as well as on the precision sought.

To obtain the values of $J_{1 n}$ and $J_{2 n}$, we express the no-slip condition at the tube wall for $u_{1}^{*}$ and we adopt a method similar to that used for $\psi_{1}^{*}$ in Eq. (29) and (30).

The obtained results show, in Figure $8(\mathrm{a})-(\mathrm{c})$, the effect of the shape of the cross-section of the tube on the azimuthal velocity distribution in the section. It can be seen that the considered geometrical shapes for $\gamma=3$ and $\gamma=5$ are generating non-symmetrical velocity distributions in contrast to the circular case.

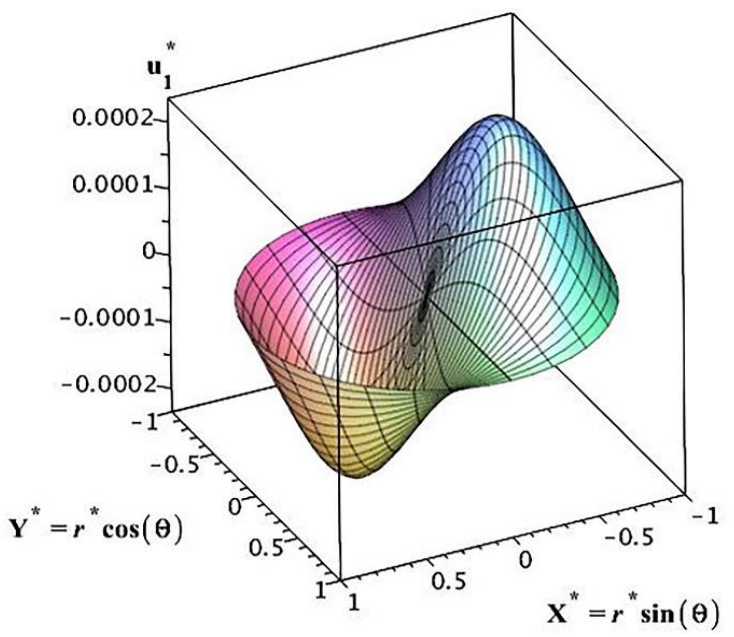

(a)

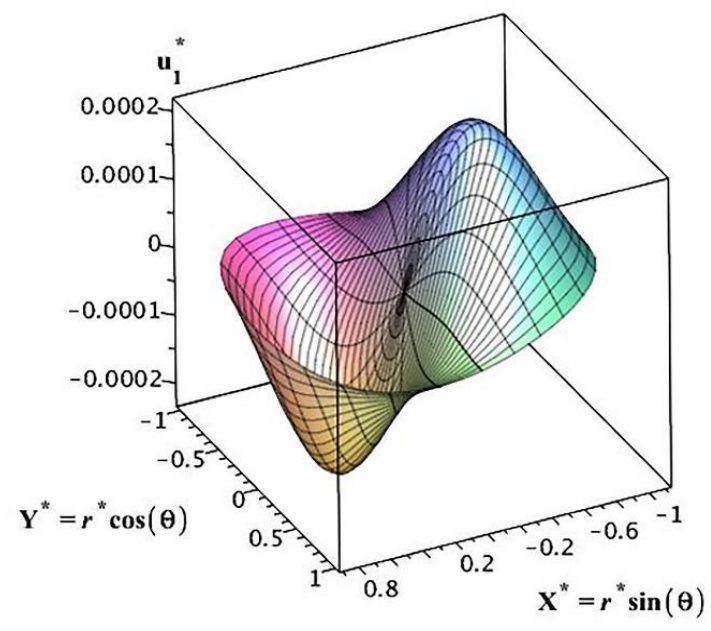

(b)

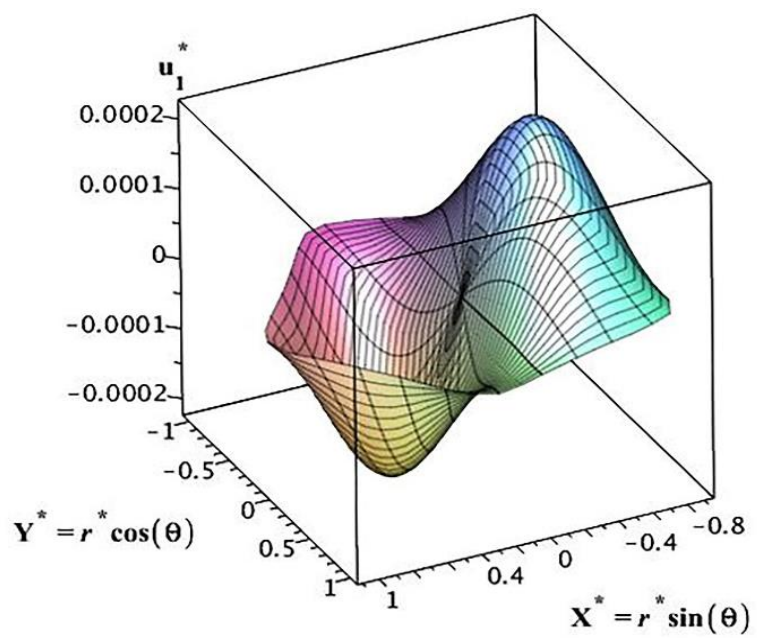

(c)

Fig. 8. The velocity distribution $u_{1}^{*}$ in $3 D$ for each section, (a): "Circular" $\gamma=0, \delta=0$, (b): "triangular" $\gamma=3, \delta=0.2$, (c): "pentagonal" $\gamma=5, \delta=0.15$ 


\section{Conclusion}

An original semi-analytical method has been proposed to solve the governing equations for the stationary motion of an incompressible Newtonian fluid in a curved pipe with various cross-sections shapes and with a small variation of curvature at different orders of $D$ number.

Solutions for the nonhomogeneous biharmonic equation for the secondary flows have been obtained by using Michel's general solution in polar coordinate for the homogeneous part and exact expressions for the particular solutions. The obtained results agree with the usual results for circular and elliptical sections cases and allow to highlight the effects related to the variation of the section shapes on the flow characteristics. Order one solutions for vortexes and azimuthal velocity are also presented. Finally, it should be noted that the original method presented here is suitable for a wide range of geometries for which explicit solutions are not available.

\section{Acknowledgment}

This work is done with the financial support of the National Center for Scientific and Technical Research (CNRST).

\section{References}

[1] Letelier, Mario F., Cristian B. Hinojosa, and Dennis A. Siginer. "Analytical solution of the Graetz problem for nonlinear viscoelastic fluids in tubes of arbitrary cross-section." International Journal of Thermal Sciences 111 (2017): 369-378.

http://doi.org/10.1016/i.ijthermalsci.2016.05.034

[2] Garg, P., J. R. Picardo, and S. Pushpavanam. "Chaotic mixing in a planar, curved channel using periodic slip." Physics of Fluids 27, no. 3 (2015): 032004. http://doi.org/10.1063/1.4915902

[3] Rasooli, Reza, Onur Kaan Karaoğlu, and Barbaros Çetin. "Simulation of Particle Separation Using Inirtial Microfluidics in a Spiral Microchannel for Biomedical Aplications." In 5th Micro and Nano Flows Conference, pp. 1114. 2016.

[4] Siggers, J. H., and S. L. Waters. "Steady flows in pipes with finite curvature." Physics of fluids 17, no. 7 (2005): 077102. http://doi.org/10.1063/1.1955547

[5] Zaho, Q., Yuan, D., Zhang, J. and Li, W. "A review of secondary flow in inertial microfluidics." Micromachines 11, no. 461 (2020): 1-23. http://doi.org/10.3390/mi11050461

[6] Martel, Joseph M., and Mehmet Toner. "Inertial focusing dynamics in spiral microchannels." physics of fluids 24 , no. 3 (2012): 032001. http://doi.org/10.1063/1.3681228

[7] Guan, Guofeng, Lidan Wu, Ali Asgar Bhagat, Zirui Li, Peter CY Chen, Shuzhe Chao, Chong Jin Ong, and Jongyoon Han. "Spiral microchannel with rectangular and trapezoidal cross-sections for size based particle separation." Scientific reports 3 (2013): 1475.

http://doi.org/10.1038/srep01475

[8] Berger, S. A. and Talbot, L. "Flow in curved pipes." Ann. Rev. Fluid Mech. 15 (1983): 461-512.

[9] Yanase, S. Mondal, R. N. and Kaga, Y. "Numerical Study of Non- isothermal Flow with Convective Heat Transfer in a Curved Rectangular Duct." Int. J. Thermal Sciences 44 (2005): 1047-1060. http://doi.org/10.1016/i.ijthermalsci.2005.03.013

[10] Mondal R. N., Islam M. Z., Islam M. M., Yanase S. "Numerical Study of Unsteady Heat and Fluid Flow through a Curved Rectangular Duct of Small Aspect Ratio." Thammasat International Journal of Science and Technology, 20 no. 4 (2015): 1-20.

[11] Norouzi, M., and N. Biglari. "An analytical solution for Dean flow in curved ducts with rectangular cross section." Physics of Fluids 25, no. 5 (2013): 053602.

http://doi.org/10.1063/1.4803556 
[12] Mahrous, Samar Ahmed, Sidik, Nor Azwadi Che, and Saqr, Khalid Mansour. "Investigation of Newtonian and Pawerlaw blood flow mpdels in $180^{\circ}$ curved pipe at low to medium shear rate." Journal of Advanced Research in Fluid Mechanics and Thermal Sciences 69, no. 1 (2020): 148-162.

https://doi.org/10.37934/arfmts.69.1.148162

[13] Dean, Wo R. "XVI. Note on the motion of fluid in a curved pipe." The London, Edinburgh, and Dublin Philosophical Magazine and Journal of Science 4, no. 20 (1927): 208-223. http://doi.org/10.1080/14786440708564324

[14] Dean, W. R. "The streamline motion of fluid in a curved pipe." Phil. Mag. 5 (1928): 673-693. http://doi.org/10.1080/14786440408564513

[15] Eustice, John. "Flow of water in curved pipes." Proceedings of the Royal Society of London. Series A, Containing Papers of a Mathematical and Physical Character 84, no. 568 (1910): 107-118.

http://doi.org/10.1098/rspa.1910.0061

[16] Eustice, John. "Experiments on stream-line motion in curved pipes." Proceedings of the Royal Society of London. Series A, Containing Papers of a Mathematical and Physical Character 85, no. 576 (1911): 119-131. http://doi.org/10.1098/rspa.1911.0026

[17] Cuming, H. G. The secondary flow in curved pipes. No. 2880. HM Stationery Office, 1952.

[18] Ito, Hidesato. "Theory on laminar flow through curved pipes of elliptic and rectangular cross-section." Rep. Inst. High Speed Mech., Tohoku Univ. Sendai Japan 1 (1951): 1-16.

[19] Collins, W. M., and S. C. R. Dennis. "The steady motion of a viscous fluid in a curved tube." The quarterly journal of mechanics and applied mathematics 28, no. 2 (1975): 133-156. http://doi.or/10.1093/qjmam/28.2.133

[20] Wang, C. Y. "On the low-Reynolds-number flow in a helical pipe." Journal of Fluid Mechanics 108 (1981): 185-194.. http://doi.org/10.1017/s0022112081002073

[21] Germano, M. "On the effect of torsion on a helical pipe flow." Journal of Fluid Mechanics 125 (1982): 1-8. http://doi.org/10.1017/s0022112082003206

[22] Gammack, David, and Peter E. Hydon. "Flow in pipes with non-uniform curvature and torsion." Journal of fluid mechanics 433 (2001): 357-382. http://doi.org/10.1017/s0022112001003548

[23] Pedley, T. J. "The fluid mechanics of large blood vessels: Cambridge Univ." (1980). http://doi.org/10.1017/CBO9780511896996

[24] Zakaria, Mohamad Shukri, Farzad Ismail, Masaaki Tamagawa, Ahmad Fazli Abdul Azi, Surjatin Wiriadidjaya, Adi Azrif Basri, and Kamarul Arifin Ahmad. "Computational fluid dynamics study of blood flow in aorta using OpenFOAM." Journal of Advanced Research in Fluid Mechanics and Thermal Sciences 43, no. 1 (2018): 81-89.

[25] Fellouah, H., C. Castelain, A. Ould El Moctar, and H. Peerhossaini. "A criterion for detection of the onset of Dean instability in Newtonian fluids." European Journal of Mechanics-B/Fluids 25, no. 4 (2006): 505-531. http://doi.org/10.1016/j.euromechflu.2005.11.002

[26] Timité, Brahim, Cathy Castelain, and Hassan Peerhossaini. "Pulsatile viscous flow in a curved pipe: Effects of pulsation on the development of secondary flow." International Journal of Heat and Fluid Flow 31, no. 5 (2010): 879-896. http://doi.org/10.1016/j.ijheatfluidflow.2010.04.004

[27] Islam, M.Z., Mondal, R. N. and Rashidi, M. M. "Dean-Taylor flow with convective heat transfer through a coiled duct." Computers \& Fluids 149 (2017): 41-55.

http://doi.org:10.1016/i.compfluid.2017.03.001

[28] Masliyah, Jacob H. "On laminar flow in curved semicircular ducts." Journal of Fluid Mechanics 99, no. 3 (1980): 469479.

http://doi.org/10.1017/s0022112080000717

[29] Lekner, John. "Viscous flow through pipes of various cross-sections." European journal of physics 28, no. 3 (2007): 521. http://doi.org/10.1088/0143-0807/28/3/014

[30] LETELIER S, M. F., and Hans J. Leutheusser. "Laminar flow in conduits of unconventional shape." Journal of engineering mechanics 111, no. 6 (1985): 768-776. http://doi.org/10.1061/(asce)0733-9399(1985)111:6(768)

[31] Mortensen, Niels Asger, Fridolin Okkels, and Henrik Bruus. "Reexamination of Hagen-Poiseuille flow: Shape dependence of the hydraulic resistance in microchannels." Physical Review E 71, no. 5 (2005): 057301. http://doi.org/10.1103/physreve.71.057301

[32] Lai, W.M., Rubin, D. and Krempl, E. Introduction to continuum mechanic: $3^{\text {rd }}$ Ed., Butterworth-Heinemann, 1999. 
[33] Chen, Guo, Zhilin Li, and Ping Lin. "A fast finite difference method for biharmonic equations on irregular domains and its application to an incompressible Stokes flow." Advances in Computational Mathematics 29, no. 2 (2008): 113-133.

http://doi.or/10.1007/s10444-007-9043-6

[34] Taylor, Cedric, and Paul Hood. "A numerical solution of the Navier-Stokes equations using the finite element technique." Computers \& Fluids 1, no. 1 (1973): 73-100. http://doi.org/10.1016/0045-7930(73)90027-3

[35] Young, D. L., C. W. Chen, C. M. Fan, K. Murugesan, and C. C. Tsai. "The method of fundamental solutions for Stokes flow in a rectangular cavity with cylinders." European Journal of Mechanics-B/Fluids 24, no. 6 (2005): 703-716. http://doi.org/10.1016/i.euromechflu.2005.03.003

[36] Canton, Jacopo, Ramis Örlü, and Philipp Schlatter. "Characterisation of the steady, laminar incompressible flow in toroidal pipes covering the entire curvature range." International Journal of Heat and Fluid Flow 66 (2017): 95-107. http://doi.org/10.1016/i.ijheatfluidflow.2017.05.014 